\title{
LA DIALÉCTICA PLATÓNICA Y EL PARMÉNIDES
}

\author{
ÁLVARO VALLEJO CAMPOS \\ Universidad de Granada
}

\begin{abstract}
RESUMEN: El autor examina las líneas de continuidad y las diferencias que existen respecto a la dialéctica platónica entre el Fedón y la República, por un lado, y el Parménides y el Teeteto, por otro, ya que solo en estos últimos y especialmente en el Parménides es donde podemos encontrar una demostración del método. Las reflexiones metodológicas del Parménides sobre la dialéctica insisten en tres dimensiones: (a) un componente gimnástico en el que debe ejercitarse el pensamiento, (b) la necesidad de explorar caminos que muestran la inviabilidad o la errancia de las tesis ontológicas propuestas y (c) su indudable conexión con la verdad. Dada la diversidad de interpretaciones suscitadas por las ocho hipótesis de la segunda parte del Parménides, el autor explora el vocabulario cognitivo que contienen como un indicio muy claro del valor de verdad o de la inviabilidad que Platón concedía a cada una de ellas, lo cual pone de manifiesto cómo se ejecuta la metodología dialéctica a la que tiene que atenerse el pensamiento filosófico.
\end{abstract}

PALABRAS CLAVE: Platón; dialéctica; vocabulario cognitivo; hipótesis; Fedón; República; Parménides; Teeteto.

\section{Plato's dialectic and the Parmenides}

ABSTRACT: The author examines the lines of continuity and the differences in relation to Plato's dialectic between the Phaedo and the Republic, on the one hand, and the Theatetus and the Parmenides, on the other, for it is only in the latter where we find a demonstration of the dialectic exercise. The methodological reflexions on dialectic in the Parmenides establish in his view three dimensions: (a) a gymnastic component which is necessary for thought, (b) the need to explore ways that show the impracticability or the wandering of the ontological theses proposed and (c) its undeniable connexion with truth. Given the great diversity of interpretations that have been proposed about the validity of the eight hypotheses in the second part of the Parmenides, the author tries to clarify the true value of the hypotheses or their impracticability exploring the cognitive vocabulary within them, because this procedure reveal how has to be executed.

KEY WORDS: Plato; dialectic, cognitive vocabulary; hypothesis, Phaedo; Republic; Parmenides; Theaetetus.

\section{INTRODUCCIÓN}

El propósito fundamental de este trabajo es intentar averiguar cómo entendía Platón los procedimientos discursivos de la dialéctica, pues esta representaba según su declaración expresa la culminación del pensamiento racional. La dialéctica es una práctica que se hace necesaria especialmente en los debates y controversias filosóficas, pero que se propone también como pauta necesaria del razonamiento, de una manera que no carece de aplicaciones en el presente. El Teeteto y el Parménides son dos diálogos enigmáticos, que, según los análisis estilométricos más acreditados, se escribieron después de la República y antes de los últimos diálogos de madurez, en los que Platón ensayó un modelo de dialéctica basado en la reunión y la división, donde el objetivo fundamental parece consistir en la definición de las ideas y el establecimiento de sus mutuas relaciones. Comentar un texto no consiste en otra cosa que en interpretarlo y 
esclarecer el sentido de sus palabras. En el caso del Teeteto, el tema propuesto es el conocimiento, una cuestión, como es sabido, conectada esencialmente en el Fedón y la República con la teoría de las formas, y, sin embargo, estas parecen estar ausentes en los diversos ensayos de definición que se intentan en la obra, de manera que en apariencia el diálogo termina aporéticamente. Por esta razón, la incertidumbre en la interpretación se deriva de que no recibimos una respuesta positiva acerca de qué es el saber ni se dice explícitamente por qué las ideas han desaparecido del horizonte teórico inmediato cuando se trata de buscar una respuesta a la pregunta. Si nos acercamos al Parménides, anterior con toda probabilidad al Teeteto, el enigma es aún mayor, pues en este caso, al principio, la teoría de las ideas se somete a todo tipo de críticas, sin que se le dé al lector ninguna respuesta clara sobre el modo de superarlas. Después de esta primera parte y, a continuación de una sección introductoria, se plantean ocho hipótesis sobre el Uno y la multiplicidad (o nueve, al parecer de otros) que se contradicen unas a otras sin que tampoco se diga en ningún momento si son todas falsas, todas verdaderas o unas verdaderas y otras falsas. El lector comprenderá así la incertidumbre de los intérpretes, que han ofrecido los pareceres más opuestos al enfrentarse con lo que parece a primera vista un enigma indescifrable.

El propósito del presente trabajo es introducir en todo este meollo de problemas la cuestión de la concepción platónica de la dialéctica, la cual, dicho sea desde el principio, no es un asunto nada claro, pues el Fedón y, sobre todo, la República, que hablan de ella, solo esbozan un programa ideal según el cual la dialéctica es la ciencia suprema de la justificación de los principios. Pero no se dan indicaciones claras de los procedimien tos por los que se arriba dialécticamente al "principio no supuesto»

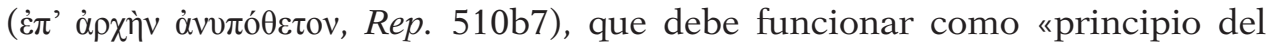

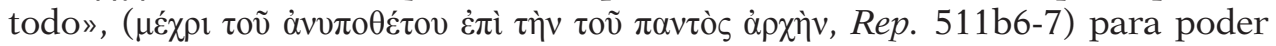
suprimir el carácter supuesto de las hipótesis con las que trabajan, por ejemplo, las disciplinas matemáticas en sus procedimientos deductivos. No es asunto del presente trabajo explicar cómo podría concebirse a ojos de Platón esta unificación de los principios por medio de los procedimientos dialécticos ${ }^{1}$. Pero Sócrates afirma en la República que, gracias a esta labor de justificación, el dialéctico es el que puede «dar razón a sí mismo y los demás» ( $\lambda$ ó

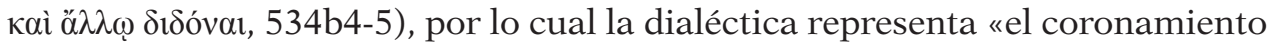

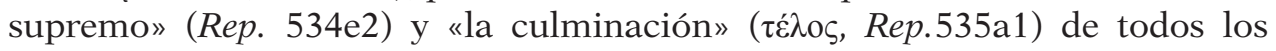
estudios previstos en la formación del filósofo gobernante.

El Teeteto y, especialmente, el Parménides, del que vamos a ocuparnos aquí con más detenimiento, nos dan unas indicaciones acerca de la dialéctica mucho menos pretenciosas que la República. Pero nos muestran, en cambio, cómo funciona efectivamente el procedimiento argumentativo que conduce a

1 Me he ocupado de ello en otro lugar: cfr. Vallejo Campos, A., Adonde nos lleve el logos. Para leer la República de Platón, Trotta, Madrid, 2018, pp. 196 y s., 226 y ss. Las citas en griego proceden de la edición de las obras platónicas debida a BuRnet, J., Platonis Opera, Oxford University Press, Oxford, 1976-1977 (reimp. de 1ª ed. 1901). 
la justificación filosófica de una hipótesis. Esta es la idea central que inspira el presente trabajo, pero, como ocurre tantas veces en la filosofía antigua, no se trata solo de una cuestión historiográfica, sino de entender una propuesta platónica acerca del funcionamiento de las justificaciones racionales que no carece de aplicaciones en el presente. Los intérpretes han tendido muchas veces a presentar estos dos diálogos como un ensayo platónico de autocrítica o, al menos, como un replanteamiento destinado a matizar algunas de sus afirmaciones anteriores, sin una relación directa de continuidad con los diálogos más dogmáticos y la idea del método dialéctico que estos últimos parecían propugnar. En relación con el Teeteto, a algunos conspicuos especialistas del s. $\mathrm{xx}$, el momento de crisis que representa esta obra en la teoría de las ideas les pareció tan genuino que se atrevieron a poner en duda la datación posterior normalmente atribuida al Timeo, ya que en esta obra se volvía a hablar de la teoría de las formas como si fuese relativamente inmune a lo dicho tanto en el Teeteto como en el Parménides. La polémica que mantuvieron a este respecto G. E. L. Owen y H. Cherniss ${ }^{2}$ llega hasta nuestros días cuando se trata de establecer el alcance de la retractación que supuestamente tuvo lugar en esos diálogos. R. Robinson, autor de la más conocida monografía sobre la dialéctica platónica en el s. xx, afirmó, por ejemplo, que el Parménides «no contiene ningún posicionamiento doctrinal, ni directa ni indirectamente»y, respecto a la segunda parte de la obra, de la que vamos a ocuparnos, sostuvo que tampoco comporta ningún pronunciamiento «respecto al método» ${ }^{3}$. En relación con la segunda parte del Parménides, en la que el sabio de Elea expone ocho hipótesis como demostración de esa «facultad dialéctica» (Parm. 135d2), que, a su juicio, hay que cultivar antes de poder defender adecuadamente la teoría de las ideas, las opiniones han sido muy dispares. Filósofos como B. Russell o G. Ryle, y otros después de ellos, han subrayado el carácter meramente antinómico de las hipótesis, de manera que cada par constituiría «una conjunción lógicamente imposible», para concluir, en opinión del segundo, que toda la segunda parte del Parménides no tiene otro propósito que «mostrar un error lógico radical» entre los presupuestos de la teoría de las formas que se exponen en la primera parte ${ }^{4}$. De manera que las cuatro parejas de hipótesis no constituirían más que «una reductio ad absurdum» ${ }^{5}$. En el otro extremo de las interpretaciones académicamente más acreditadas, C. Meinwald, autora de una famosa monografía sobre el Parménides, afirma,

2 Cfr. Owen, G. E. L., "The place of the Timaeus in Plato's Dialogues» y CHerniss, H. F., «The Relation of the Timaeus to Plato's Later Dialogues», incluidos en Allen, R. E., Studies in Plato's Metaphysics, Routledge, N. York, 1965.

3 Cfr. Robinson, R., Plato's Earlier Dialectic, Oxford University Press, Oxford, 1953, p. 223. Ya antes que él platonistas de la talla de J. Burnet o A. E. Taylor opinaban que la segunda parte del Parménides no era otra cosa que una mera parodia.

4 Cfr. Ryle, G., «Review of F. M. Cornford, Plato and Parmenides», Mind, 48 (1939), 536543, pp. 542 y s.

5 Cfr. Ryle, G., ibid., y «Plato's Parmenides», Mind, 48 (1939), 302-325, p. 305. 
por el contrario, que las contradicciones entre estas hipótesis son «meramente aparentes», por lo que, a su parecer, "podemos aceptar todos los argumentos y sus conclusiones ${ }^{6}$. Entre ambos extremos, M. L. Gill, traductora del diálogo y experta en su interpretación, defiende, sin embargo, en trabajos recientes que las dos primeras hipótesis «arrojan resultados extremos e inaceptables» ${ }^{7}$, como ocurre con la mayoría de las demás, de manera que, en realidad, el objetivo es que el lector vaya de hipótesis en hipótesis para comprobar que solo se puede salir de tales antinomias si volvemos a la primera parte de la obra y negamos el supuesto de que una forma no puede participar de las demás.

El Parménides, en resumidas cuentas, es el menos claro de todos los diálogos platónicos, no solo por las ambigüedades con las que está construido, sino por las extremas divergencias de los intérpretes. Ante este panorama, es decir, ante la incertidumbre en que a veces parecen precipitarnos tanto los argumentos empleados por el mismo Parménides como la interpretación de los especialistas, no tenemos más remedio que atender a las indicaciones que Platón nos va dando acerca de sus intenciones, pues interpretar un texto, como hemos dicho, es averiguar el sentido al que apuntan sus palabras. Lo mejor, desde luego, sería llegar a unos resultados concluyentes considerando solo un examen cuidadoso de los argumentos lógicos empleados, pero, acerca de su validez dialéctica, se han pronunciado, como hemos visto, las opiniones más contrarias. Como si estuviéramos ante un tribunal, abogo, pues, por no descuidar los indicios que Platón nos da en la obra, además de toda la atención que podamos prestar a las pruebas en sí mismas. Nuestro interés radica sobre todo en la idea de la dialéctica que se pone en práctica en estos diálogos, después de lo dicho en la República, que, como hemos visto, es más bien un programa ideal que una demostración clara acerca de la metodología que hay que emplear. Las indicaciones metodológicas proporcionadas por el Parménides (a), la práctica dialéctica del Teeteto (b) y, sobre todo, las hipótesis que se desarrollan a título de ejemplo en la segunda parte del Parménides (c) pueden sernos útiles si queremos entender no solo los procedimientos dialécticos contemplados en la República, que no quedan suficientemente precisados en esa obra, sino también la dialéctica que se desarrollaba en la Academia a la hora de enfrentarse a tesis filosóficas ajenas o tener que defender las propias.

\section{Reminiscencias de la República y el Fedón}

Antes de lidiar con el Parménides, me propongo recordar al lector algunas indicaciones muy útiles que se encuentran tanto en el Fedón como en la p. 23.

6 Cfr. Meinwald, C., Plato's Parmenides, Oxford University Press, Oxford-N.York, 1991,

7 Cfr. Gill, M. L., «Design of the Exercise in Plato's Parmenides», en Dialogue 53 (2014), 495-520, p. 503; cfr. también, Philosophos: Plato's Missing Dialogue, Oxford University Press, Oxford, 2012, pp. 45-75. 
República acerca de la justificación de los principios. En la primera de estas dos obras, Sócrates nos previene del peligro de la misología, que es el odio que puede generarse contra los argumentos, cuando, por una falta de pericia ( $\dot{\alpha} \tau \varepsilon \chi^{\prime \prime} \alpha$, cfr. Fed. 90d3), perdemos la confianza en la verdad que por medio de ellos se puede alcanzar. Quisiera destacar la íntima relación que se establece en estos pasajes preliminares ( $F e d$. 89d1-91b1) entre tres términos que son fundamentales para nuestro tema: los argumentos (lógoi), la verdad y la técnica que debe presidir

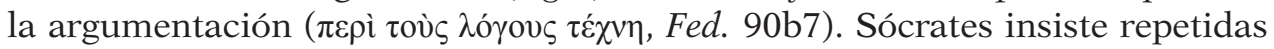

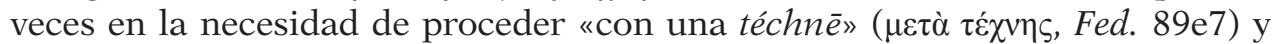
de no intentar defender o atacar un argumento «desprovisto del arte» de los

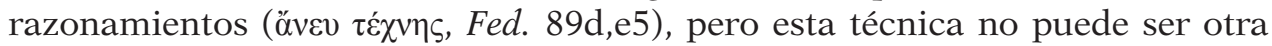
que la dialéctica. Su competencia es la misma que aquella a la que se refiere Sócrates en el Teeteto cuando describe "la técnica» de su mayéutica ( $\tau \tilde{\eta} \varsigma \dot{\varepsilon} \mu \tilde{\eta} \varsigma$ $\tau \dot{\varepsilon} \chi \nu_{\eta} \varsigma \tau \tilde{\eta} \varsigma \mu \alpha \iota \varepsilon v \tau 1 \kappa \tilde{n} \varsigma$, Teet. 150b6; $\mathrm{c1}^{8}$ ): es la que hay que emplear para probar si la doctrina defendida por un joven interlocutor es «algo imaginario y falso o fecundo y verdadero» (Teet. 150c1-3). El Sócrates de Platón que aparece en esta obra, sin duda tardía, insiste en el hecho de que él posee este «arte» (Teet. 149a7). Su conexión con la verdad es tal que, si Protágoras estuviera en lo cierto y cada uno fuera medida de todas las cosas, según declara Sócrates, «la totalidad de la

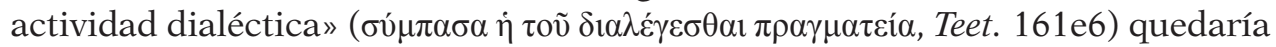
puesta en ridículo, porque no serviría a su finalidad fundamental, que consiste en el esclarecimiento de la verdad.

Volvamos al Fedón. Después de esta presentación, en la que se insiste en la necesidad de proceder con una técnica de los argumentos para no caer en la misología, Sócrates nos describe sumariamente los procedimientos de esta téchnē, que constituyen el núcleo de la dialéctica tal como Platón la concebía en esta época. Es el método de las hipótesis (cfr. Fed.100a-101d), porque la dialéctica consiste en una práctica argumentativa que permite dar razón ( $\delta 1 \delta o ́ v a ı ~ \lambda o ́ \gamma o v$, Fed. 101d6) de una tesis filosófica examinando, por un lado, las consecuencias deductivas que se derivan de ella y, por otro, su propia concordancia con otros principios situados «por encima» ( $\alpha \omega \omega \theta \varepsilon v, 101 \mathrm{~d} 6)$ en la cadena deductiva en la que ella misma debe insertarse. Como hemos dicho en otro lugar ${ }^{9}$, la dialéctica no tiene nada que ver con un misticismo especulativo que se basara en una intuición destinada a la captación inmediata de la verdad, sino con unos procedimientos deductivos en los que se trata de examinar las consecuencias que se derivan de la adopción de una hipótesis. El punto central del método, según lo aclara el propio Platón, consiste en distinguir entre la hipótesis ( las consecuencias que se desprenden de él (Fed.101e2-3), por otro. Se anuncia,

8 Sócrates insiste en el Teeteto en el carácter técnico de la dialéctica que practica con su mayéutica; cfr. Teet. 149a4, a7, 150b6, 161e5, 184b1, 210b8, c4.

9 Cfr. Vallejo Campos, A., o.c., p. 32, 227 y 233; véase también «La intuición, el programa dialéctico de la República y su práctica en el Parménides y el Teeteto », Plato Journal, 20 (2020), 137-150, pp. 141-144. 
en concordancia con la República ${ }^{10}$, la existencia de un doble procedimiento, ascendente y descendente. Igual que en esta otra obra, la metáfora del arriba y abajo, referida a otras proposiciones con las que la hipótesis está relacionada, no puede tener otro significado que la posición respectiva que guardan entre sí los principios en el sistema deductivo del que forman parte. Hacia abajo el examen dialéctico consiste en extraer consecuencias, para comprobar si «las

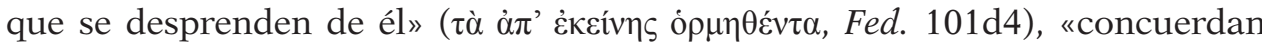

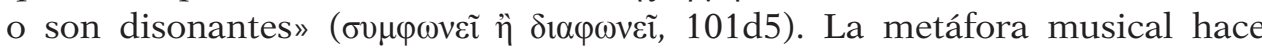
referencia aquí a la relación de compatibilidad o incompatibilidad lógica que puede existir entre las proposiciones derivadas de la hipótesis en cuestión. Si de un principio adoptado, como punto de partida en una discusión filosófica, se siguieran consecuencias contradictorias o inaceptables desde el punto de vista filosófico propuesto, el examen dialéctico conduciría naturalmente al rechazo de la hipótesis. En caso contrario, debe seguirse un proceso ascendente para mostrar la relación lógica en la que se halla el principio objeto de debate con otro del que puede derivarse deductivamente, por su mayor universalidad o porque es claramente anterior en el proceso de inferencia. Aquí no se dice, como en la República, que el proceso dialéctico deba tomar las hipótesis como "peldaños y trampolines» hasta alcanzar «un principio del todo, que es no supuesto» (Rep. 511b6-7), interpretado por la mayoría de los especialistas como la idea del Bien, sino que, dado el carácter meramente metodológico del pasaje, se habla solo de remontarse hasta otro principio «que parezca mejor de

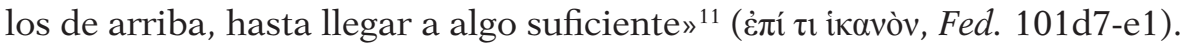

Si nos fijamos ahora en el momento descendente de la prueba dialéctica a la que tiene que someterse la discusión de un principio, es evidente que se contemplan dos posibilidades, una positiva, a favor de su admisión provisional a la vista de los resultados «concordantes» derivados de ella, pero también otra negativa, que impone su rechazo cuando los resultados deductivos son inadmisibles por su «disonancia». Esta doble posibilidad contemplada en el Fedón es la que cabría esperar naturalmente de la discusión dialéctica, si tenemos en cuenta la diversidad y la contradicción de posiciones teóricas que concurren en las discusiones filosóficas, tanto en el pasado como en el presente. Tengamos en cuenta este hecho cuando examinemos la dialéctica del Parménides. En el examen socrático, característico de los diálogos iniciales, predomina, como es sabido, el momento elénctico, que se derivó de la enigmática respuesta dada por

10 Sobre este punto, cfr. Vallejo Campos, A., o.c., p. 197. Para las diferencias y semejanzas entre las prácticas dialécticas previstas en el Fedón y la República, cfr. RoBinson, R., o.c., p. 170-171.

11 Cfr. Hackforth, R., Plato's Phaedo, Liberal Arts Press, Cambridge-New York, 1952, p. 141. Sin embargo, a favor de conectar este pasaje del Fedón con la idea del Bien, que es en la República la culminación del proceso dialéctico, cfr., p.e., Eggers Lan, C., El Fedón de Platón, Eudeba, Buenos Aires, 1993, p. 189. 
el oráculo a la pregunta que le formuló su amigo Querefonte ${ }^{12}$. Se trataba ante todo de eliminar las certezas infundadas que poseían los ciudadanos con los que Sócrates se encontraba en una Atenas soliviantada por pasiones desmedidas. Pero, para una construcción deductiva de la ciencia, en la que se habla, por ejemplo, de fundamentar hipótesis matemáticas, como se hace en la República, la justificación racional no puede consistir solo en la contradicción de una tesis. La dialéctica debe aspirar al momento de una construcción positiva, aludido en la recreación tardía que hace Platón de la dialéctica socrática cuando afirma, en el Teeteto, que está destinada a mostrar si lo que contienen los pensamientos del interlocutor es algo «algo imaginario y falso o fecundo y verdadero» (Teet. 150c1-3).

La República pone todo su énfasis en el momento positivo y en el ascenso, porque este conduce con toda seguridad a una unidad de todas las ciencias fundada en la cobertura ontológica que les presta la idea del Bien. Sin embargo, también aquí está fuertemente presente, en mi opinión, el momento negativo que es connatural a toda dialéctica, pues en uno de los momentos decisivos en los que se trata precisamente de definir al dialéctico como aquel que es capaz de «alcanzar la razón de la esencia» (Rep. 534b3-4), se dice, a propósito del Bien, que es necesario «atravesar todas las refutaciones como en medio de

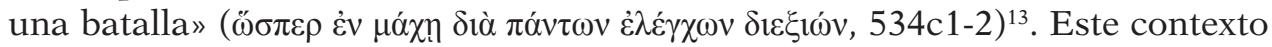
agonal y refutatorio es donde la dialéctica tiene que moverse, sobre todo cuando se trata de una confrontación entre tesis filosóficas opuestas. Tal parece ser de hecho el escenario al que Platón ha querido trasladarla cuando pasamos de la República al Parménides y al Teeteto, antes de mostrarnos en diálogos sucesivos su cara más positiva, que la presenta como instrumento para el esclarecimiento de las mutuas relaciones entre las ideas. En la lectura unitaria que propongo de los diálogos no se trata de negar las posibles diferencias que haya en los planteamientos de cada uno, sino únicamente de subrayar sus líneas de continuidad.

En la práctica platónica de la dialéctica se conjugaban con toda probabilidad estos dos momentos, positivo y negativo, si queremos llamarlos así, o constructivo y refutatorio. En el Crátilo (390c10-11) se dice que llamamos dialéctico al «que

12 Cfr. Vallejo Campos, A.-Vigo, A., Filósofos griegos: de los sofistas a Aristóteles, Eunsa, Pamplona, 2017, pp. 117 y ss.

13 E. BERTI ha subrayado el papel que este texto le atribuye a la refutación en los procedimientos de la dialéctica en la República, en la que a primera vista el élenchos no parece tener ningún papel. Cfr. Berti, E., Nuovi studi aristolici, Morcelliana, Brescia, 2004, pp. 344-5, 361, 421. Sin embargo, esto no significa, a mi juicio, que la dialéctica platónica consista solo «en el arte de pedir y dar razón por medio de la refutación» (ibíd.., p. 421),

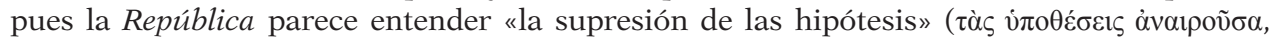
$533 \mathrm{c} 8$ ) como un procedimiento que puede ser también positivo, ya que en el caso de las hipótesis matemáticas no se trata de su eliminación sino más bien de una fundamentación racional que «suprima» su carácter hipotético. Sin embargo, como se verá a continuación, mi coincidencia con el Prof. Berti en lo referente al Parménides es enorme. Cfr., en este sentido, Vallejo Campos, A., Adonde nos lleve el logos, p. 229 y, especialmente, n. 47. 
sabe preguntar y responder» y, de acuerdo con ello, el programa educativo de la República sostiene que dicha capacidad debe estimularse «máximamente» (Rep. 534d8) en la formación del futuro gobernante, pues la dialéctica, efectivamente, representa un «saber» cuya característica principal consiste en "dar razón

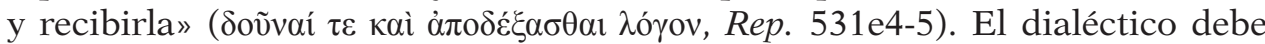
proponer, como hemos visto, una definición, es decir, un lógos tês ousías (Rep. 534b3-4), ya sea del Bien o de cualquier otra materia, y no se puede descartar que lo haga por medio de una cierta intuición noética que le permita, como dice el Fedón, elegir «la hipótesis que parezca mejor de las de arriba» (Fed. 101d7). Sin embargo, el término «intuición» puede resultar desorientador si lo entendemos como una operación del intelecto desligada de su andamiaje discursivo. La definición, de la que también trata la República (532a7, 533b2), cualquiera que sea el procedimiento con el que se ha alcanzado, forma parte de la justificación racional, que es el aspecto que prevalece en esta obra, pues el que ofrece una definición debe estar en condiciones de dar las razones por las que se atribuye a la cosa en cuestión tales o cuales propiedades. El dialéctico

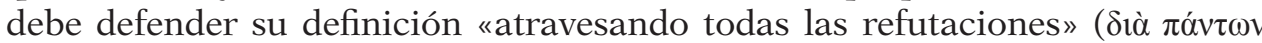

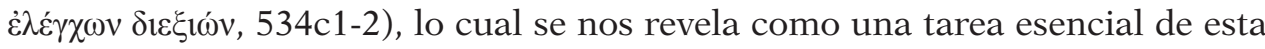
práctica, que forma parte de su obligación dialógica de dar razón y recibirla. El autor de la Carta VII parece insistir en este carácter bifronte de la práctica dialéctica que debe asumirse en el contexto de los debates filosóficos. Según lo que se dice aquí, lo que se ha entendido tradicionalmente como intuición no parece estar totalmente ausente de los procedimientos dialécticos, cuando se insiste en que, en un determinado momento, «brilla de pronto la razón y la intelección sobre cada cosa» (Carta VII 344b7), pero, a diferencia de lo que podríamos pensar por las connotaciones modernas del término, este instante forma parte sin solución de continuidad de un proceso dialógico en el que hay «que servirse de preguntas y respuestas» y someterse a «refutaciones y críticas»

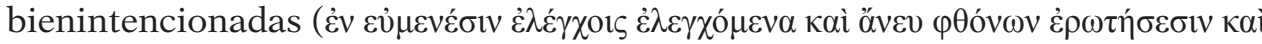

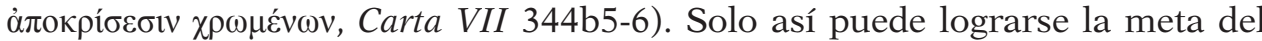
filósofo, que aparece en este testimonio como un aprendizaje de todo aquello que «es falso y verdadero en la entera realidad» (ibid., 344b2). Quedémonos con esta última frase, que parece insistir en estas dos caras, la verdad y la falsedad, como las dos metas que el ejercicio del pensamiento filosófico debe desentrañar en sus múltiples andaduras.

\section{La metodología dialéctica del PaRMÉNIDES}

En esta enigmática obra encontramos toda una reflexión sobre la dialéctica y, lo que es más importante, a mi juicio, una práctica del método que el Fedón y la República solo se limitaron a esbozar. Digo «a mi juicio», porque se trata de una interpretación que no es evidente a primera vista, pues muchos ven en el Parménides un diálogo crítico que marca una ruptura con lo anterior. El enigma 
que representa la obra fue creado, desde luego, por el propio Platón, al dedicar la primera parte a formular toda una batería de objeciones contra la teoría de las ideas, sin explicar en ningún momento cómo salir de ellas. El efecto dramático ha sido tan logrado que, como hemos visto, muchos intérpretes la han tomado como una rectificación de la teoría destinada a abandonarla o a modificarla sustancialmente. Sin embargo, en esta ocasión, nuestro tema es la dialéctica y no la teoría de las formas, y, aunque sea difícil separarlas, la cuestión es que Parménides apunta claramente a la metodología dialéctica cuando se trata de ofrecer un diagnóstico filosófico de las dificultades no resueltas por este joven e inexperto Sócrates que propone la teoría. No es nada casual en el arte dramático de Platón que haya elegido como ponente de su doctrina a un Sócrates, al que «la filosofía no lo ha atrapado aún, como lo hará más adelante» (Parm. 130e2-3). La situación en la que se halla, después de haberse sometido al examen de Parménides, es la misma en la que se han encontrado muchas veces los lectores de la primera parte: no ve ningún camino por dónde tirar (Parm. 135c7). Pero Parménides ofrece un diagnóstico del problema: el error ha consistido en "empeñarse en definir lo bello, lo justo, lo bueno y cada una de las formas», "antes de ejercitarse» ( $\pi \rho i v ~ \gamma v \mu v \alpha \sigma \theta \tilde{\eta} v \alpha 1,135 c 8)$ en esa práctica que algunos, como dice Isócrates (cfr. In soph. 8, Ant. 262), califican como mera

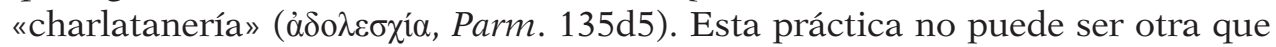
la dialéctica, a la que, por otra parte, ha aludido el mismo Parménides unas líneas antes cuando ha advertido a Sócrates de que, si desfallece y abandona las

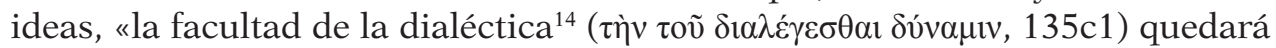
destruida» y no habrá adonde "dirigir el pensamiento» (135b8). Platón no ha podido darnos una indicación más clara que esta para saber a qué atenernos respecto a su opinión sobre la teoría que se acaba de establecer. Me limito a formular mi opinión, sin entrar a discutir otros puntos de vista sobra la teoría misma que nos apartarían de nuestro camino: esta no puede ser rechazada, pero debe ser reformulada, una vez que se hayan atendido las deficiencias dialécticas detectadas por Parménides. De manera que el sentido del diálogo, respecto a las intenciones de Platón, me parece claro: la teoría de las ideas no puede abandonarse y, de hecho, volverá a defenderse en diálogos posteriores. Pero hay que prestar atención a requisitos y refinamientos dialécticos que no fueron observados suficientemente en el momento de formularla en diálogos más dogmáticos como el Fedón y la República.

De manera que el Parménides nos ofrece para salir del paso dos cosas a partir de aquí: unas reflexiones metodológicas acerca de la dialéctica en sí misma (135c-136e) y "una demostración» (136e7) de cómo debe aplicarse en la práctica. Sería ridículo pensar que Platón, como han sugerido muchos

14 Aunque no aparece la palabra dialéctica, estoy de acuerdo con la versión que ofrece aquí M. I. SANTA CRUZ, cuya traducción del Parménides sigo a menos que indique lo contrario. Cfr., en este mismo sentido, Gill, M. L., Philosophos: Plato's Missing Dialogue, pp. 18-19, n.1, que nos recuerda la aparición de la misma expresión en Filebo 57e6-7, donde se refiere inequívocamente a la dialéctica. 
intérpretes, haya escrito toda esta última parte como un mero ejercicio sin implicaciones teóricas para resolver los problemas ontológicos que han metido al joven Sócrates en un callejón sin salida. Vayamos a ambos aspectos de la cuestión. Las reflexiones metodológicas a las que me refiero son de una importancia inestimable, ya que contienen nada menos que un programa del ejercicio de la dialéctica, pero no un proyecto idealizado como el que se evoca en la República sin que se ofrezca nunca una indicación clara de su práctica, sino una explicación anticipada de lo que el mismo Parménides se va a encargar de realizar en la última parte de la obra. Dichas reflexiones se plantean desde una triple perspectiva, cuyos términos debemos tener muy presentes para no interpretar el ejercicio dialéctico de un modo unilateral, como ha sido tan frecuente.

La primera indicación que nos da Parménides, repetida con la misma terminología hasta en cinco ocasiones $(135 \mathrm{c} 8, \mathrm{~d} 4, \mathrm{~d} 7,136 \mathrm{a} 2, \mathrm{c} 5)$, es que la dialéctica va unida a la necesidad de practicar un ejercicio ( $\gamma v \mu v \alpha \sigma i ́ \alpha$, Parm.135d7) que Sócrates debe vincular a ese «impulso que lo arrastra hacia los argumentos» (135d3). Es muy interesante, para confirmar el contraste, en cierto sentido paradójico, en el que vengo insistiendo entre la República y el Parménides, que reparemos en las diferencias entre ambos diálogos en un punto muy concreto. En la primera de estas obras, cuando Glaucón pregunta a Sócrates por el modo de proceder ( $\tau$ ó $\pi$ os) del poder dialéctico, en qué clases ( $\varepsilon \Uparrow \delta \eta)$ se divide y cuáles son sus caminos (óooí, Rep. 532d3-e1), Sócrates se niega a contestar con el pretexto de que entonces no sería capaz de seguirlo. Sin embargo, aquí el Sócrates joven e inexperto del Parménides, dado que están en un contexto literario muy diferente, puede preguntar por el modo ( $\tau \rho{ }^{\prime} \pi \mathrm{s}$, Parm.135d7) de este ejercicio que el maestro le recomienda. La primera indicación se da por medio de una remisión al procedimiento empleado por Zenón, del que este habla en las páginas introductorias del diálogo. Aquí la dialéctica funciona por reducción al absurdo: se trata de partir de una hipótesis,

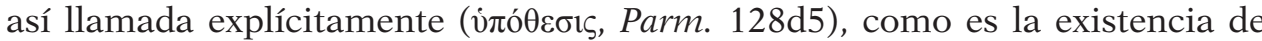
la multiplicidad, que se formula como el antecedente de un condicional («si hay multiplicidad»), para extraer de ella «consecuencias» que se consideran

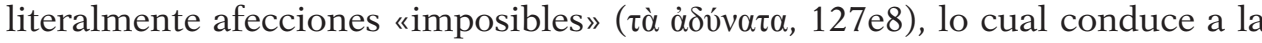
negación del supuesto del que se había partido. Tales consecuencias inadmisibles consisten en afirmar de aquellas cosas postuladas como múltiples predicados que se contradicen mutuamente (que son semejantes y desemejantes), lo cual

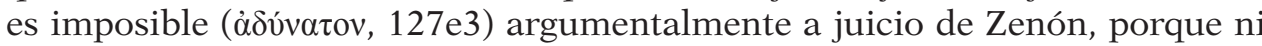
las cosas desemejantes pueden ser semejantes ni las semejantes desemejantes. El argumento es concebido como un instrumento esencial al servicio del contexto agonal y dialógico característico de las discusiones filosóficas. Lo que Zenón persigue, efectivamente, es una argumentación que constituya una

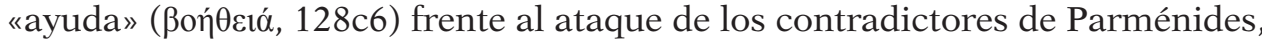
que habían ridiculizado la unidad del ser defendida por este. La ayuda consiste, lógicamente, en reducir al absurdo la tesis que se opone contradictoriamente a la concepción filosófica puesta en cuestión. Sócrates comprende perfectamente 
el efecto dialéctico de este procedimiento, que pasará a llamarse ordinariamente una prueba indirecta, pues, mientras uno (Parménides) afirma la unidad, el otro (Zenón) acude en su ayuda negando la multiplicidad, lo cual, según se afirma explícitamente, equivale a decir exactamente lo mismo ( $\lambda \dot{\varepsilon} \gamma o v \tau \alpha \varsigma \tau \alpha \dot{\tau} \tau \dot{\alpha}$, 128b5).

Pero al procedimiento de Zenón, Parménides añade dos precisiones fundamentales. La primera es que esta técnica debe emplearse no solo en el dominio de las cosas "visibles», como hacía Zenón, sino también en el caso de aquellas otras, como las formas ( $\varepsilon i \delta \eta, 135 \mathrm{e} 3)$, que solo pueden aprehenderse por medio de la razón. Me parece que esta es una observación muy pertinente, porque pone de manifiesto que lo que se nos va a describir es en realidad el método que debe emplearse para la dirección del buen uso del entendimiento. Sin embargo, la segunda precisión añade a la práctica de Zenón un factor esencial, que da carta de naturaleza a los procedimientos discursivos que son característicos de la dialéctica como instrumento racional por excelencia. Parménides afirma que, para un mayor «entrenamiento» ( $\gamma v \mu v \alpha \sigma \theta \tilde{\eta} v \alpha 1,136 \mathrm{a} 2)$, no solo debe suponerse que una cosa es y «examinar las consecuencias que se

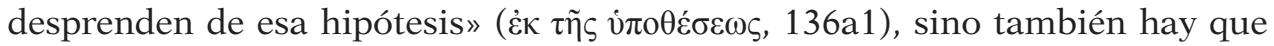
suponer que esa misma cosa no es.

Es muy interesante tener en cuenta esta recomendación cuando pasamos del Parménides al Teeteto. La parte positiva de la hipótesis platónica, por llamarla así de acuerdo con la terminología que impera en la República y el Parménides, ya había sido defendida filosóficamente en la primera de estas dos obras, donde se sostuvo la existencia de un conocimiento racional cuyo verdadero objeto son las ideas. En el Teeteto, se aplica, en mi opinión ${ }^{15}$, la recomendación dialéctica del Parménides, porque lo que en aquel diálogo comparece es justamente la tesis que se opone contradictoriamente a lo sostenido en la República. Teeteto define el conocimiento como percepción, pero Protágoras, con su conocida sentencia del hombre medida, y Heráclito, al que se le atribuye la doctrina del flujo universal, proporcionan a esta definición la dimensión filosófica necesaria para representar la tesis que hay que refutar y salir así en ayuda de la defendida por Platón. El análisis dialéctico de la posición atribuida a Heráclito y a Protágoras conduce, efectivamente, a una contradicción, pues, si «todo se mueve» podría establecerse igualmente que «las cosas son así o que no son así» (Teet. 183a). La reductio de esa posición epistemológica es tan poderosa que, a los ojos de Sócrates, no puede aceptarse «sobre la base de esa doctrina de

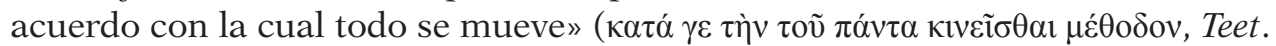
183c2-3). El lector puede aplicar al caso lo que estamos viendo en el Parménides, ya que podríamos decir que la negación del conocimiento como percepción y el rechazo de la doctrina del flujo universal en la que se apoya equivale a la

15 Me permito remitir al lector a un trabajo en el que he desarrollado esta interpretación del Teeteto, «Dialectic in the Theaetetus», en Bossi, B.-Robinson, T., Plato's Theaetetus Revisited, ed. De Gruyter, Berlín, 2020, pp. 35-50. 
defensa de la tesis filosófica sostenida en la República, según la cual hay una realidad o ousía sustraída al devenir que solo puede ser conocida por la razón. Como se dice en el Parménides, la imposibilidad, demostrada dialécticamente, de la tesis expuesta por Teeteto viene a «decir lo mismo» que lo que se ha dicho en otros diálogos acerca de la posibilidad de un conocimiento racional basado en el ser inmutable.

Voy ahora a la segunda indicación que nos da el Parménides en su parte metodológica. Este ejercicio, realizado al modo de Zenón, pero al que hay que añadirle la doble construcción hipotética, no consiste solo en una gimnástica del intelecto cuyos músculos hubiera que fortalecer antes de ponerlos al servicio de otra causa, sino que debe practicarse «para discernir bien la verdad»

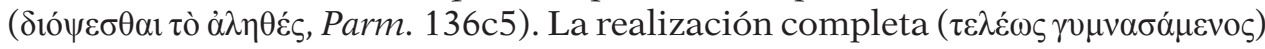
del ejercicio debe llevarse a cabo para asegurarnos de que alcanzamos «con

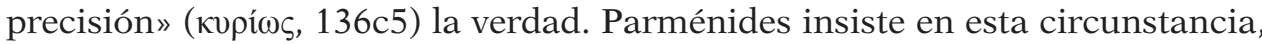
es decir, en la vinculación de la necesidad de «recorrer todos los caminos» con la posibilidad de «dar con la verdad y adquirir inteligencia de ella» (ö $\tau$ övvv

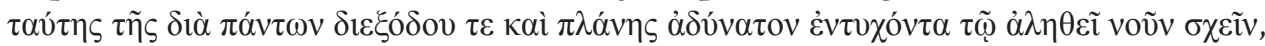
Parm.136e1-3). El vocabulario nos recuerda el pasaje antes rememorado de la República, cuando Sócrates afirmaba allí que el dialéctico, que debe estar en posesión de una razón o definición de la esencia, también está obligado a

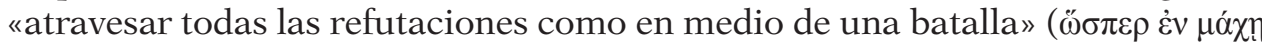

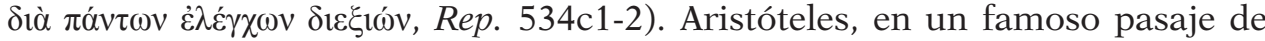
los Tópicos, en el que aborda los usos de la dialéctica, habla en primer lugar

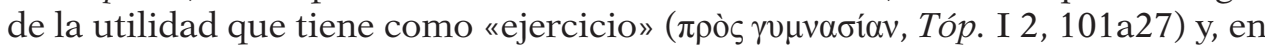
segundo lugar, además, como «método» adecuado para los meros «encuentros»

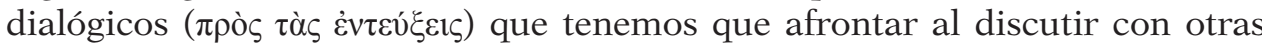
personas. Pero también nos habla, como Platón aquí, en tercer lugar, de una

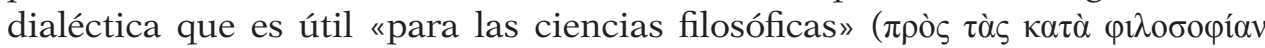

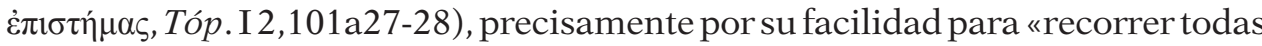
las dificultades» ( Sin poder entrar ahora en las implicaciones epistemológicas que pueda tener esta tercera utilidad de la dialéctica en la filosofía de Aristóteles, limitémonos a constatar que estos procedimientos que desarrollan un problema propuesto en ambas direcciones ( $\left.\pi \rho \hat{\jmath} \varsigma \dot{\alpha} \mu \varphi \tau_{\tau} \varepsilon \rho \alpha\right)$ son útiles precisamente porque nos permiten «fácilmente contemplar en todos los casos lo verdadero y lo falso» (Tóp. I 2, 101a35-36). El descubrimiento de la verdad y la comprobación de la falsedad que antes comentábamos son los dos caminos en los que tiene que internarse el pensamiento filosófico. La continuidad en este punto concreto entre Platón y Aristóteles es completa. Las implicaciones que tiene en relación con la verdad esta observación referente al método que se está proponiendo es indiscutible y no en balde se halla en el centro del diálogo, es decir, entre la exposición de los problemas relativos a la teoría de las ideas y la «demostración» del método que Parménides ensaya en la última parte de la obra. Desde este punto de vista, sería absurdo defender el valor de la metodología propuesta para alcanzar la verdad en las discusiones filosóficas y, sin embargo, tener que desechar toda la última 
parte dedicada al examen de las ocho hipótesis, como un ejercicio meramente antinómico del que no se puede obtener ninguna conclusión positiva. Aquí no estamos ante una mera descripción histórica del método empleado por Zenón, en defensa de Parménides, sino ante una apropiación platónica, que lo modifica para poderlo convertir en el fundamento de la práctica a seguir en las investigaciones filosóficas, cuando se trata de dirimir una controversia filosófica.

El ejercicio gimnástico defendido por Parménides no es, pues, una exigencia en sí misma desligada de los más altos intereses filosóficos, sino una preparación

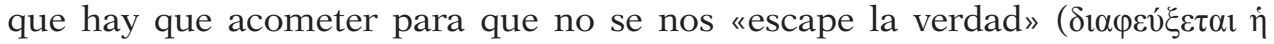
$\dot{\alpha} \lambda \dot{\eta} \theta \varepsilon \dot{\varepsilon} \alpha$, Parm. 135d6). Pero junto a estas dos indicaciones metodológicas, relativas al ejercicio y la verdad, en el pasaje antes citado (Parm. 136e) se habla, como hemos visto, de la necesidad de «recorrer todos los caminos», lo cual lleva consigo también un elemento de $\pi \lambda \alpha$ óv (Parm 136e2), es decir, de errancia o de divagación ${ }^{16}$. Se conforma, a mi juicio, así un triángulo de ideas que encierran la propuesta metodológica del Parménides: se trata (a) de recorrer todos los caminos posibles por medio de una ejercitación en el uso de los argumentos, de tal manera que, al explorarlos todos, (b) comprobaremos cuáles no conducen a ninguna parte, como le ocurre al viajero errante que no sabe adónde llegará, y (c) cuáles son verdaderos, porque estos son los caminos del pensamiento que se encuentra con el ser. He querido reunir en esta última frase esas tres palabras (camino, pensamiento y ser), de claras reminiscencias parmenídeas, porque creo que esconden una idea fundamental de la dialéctica. Esta, como se dice en la República, consiste, efectivamente, en una marcha ( $\pi$ opeía, Rep. 532b4, e3) y solo, al final de la travesía, en el «reposo del camino», se alcanza el punto donde queremos llegar (cfr. Rep. 532e2-3).

\section{El ejercicio dialéctico en la segunda parte del Parménides}

Si ahora aplicamos estas indicaciones metodológicas a la «demostración» ensayada por el sabio de Elea, hallamos a mi juicio un planteamiento que responde coherentemente a lo que se ha dicho. Nos encontramos con tesis filosóficas concebidas como hipótesis, pues el mismo Parménides nos dice que se propone indagar su «propia hipótesis» (Parm. 137b3) y averiguar las consecuencias tanto de que el Uno es como de que no es. Si dejamos a un lado, de acuerdo con muchos especialistas ${ }^{17}$, la que figura en tercer lugar como un

16 M. I. SANTA CRUZ, cuya traducción del Parménides estamos siguiendo, traduce «recorrer y explorar todos los caminos»; creo que el término lleva consigo algo más que la idea de explorar, es recorrer caminos sin estar seguros de que nos vayan a conducir adonde queremos llegar.

17 Gill, que está de acuerdo con esta consideración, da (Philosophos: Plato's Missing Dialogue, p. 49 n. 9) una lista muy completa de especialistas que han optado por esta interpretación. 
apéndice a las dos primeras, aparecen ocho hipótesis, que se construyen en pares contradictorios. Las cuatro primeras parten de la hipótesis de que lo Uno es y las otras cuatro de que no es, según el diseño estipulado por el propio Parménides en la parte metodológica del diálogo, en la que se dice que el examen de la tesis filosófica en cuestión consiste en deducir las consecuencias que se derivan tanto de la afirmación como de la negación del objeto de la hipótesis (135e-136a). Dentro de cada opción, positiva y negativa, tenemos otras cuatro hipótesis que dan las ocho resultantes, porque, según lo establecido por Parménides, hay que examinar lo que se sigue para lo Uno «respecto de sí mismo» y «respecto de los múltiples» y lo que se sigue para la multiplicidad «respecto de sí misma» y «respecto de lo Uno» (136a5-b1). Lo mismo ocurre en el caso de las otras cuatro hipótesis negativas, que se suman a las anteriores ${ }^{18}$.

No pretendo en esta ocasión entrar en el contenido ontológico de cada una de las ocho hipótesis, que queda fuera del ámbito abarcado por el presente trabajo. Tendremos que dejar ese análisis para otra ocasión, aunque es imposible no rozar el tema si lo que vamos a abordar es el valor de la verdad que Platón ha podido conceder a estas hipótesis. Si nos atenemos a la tríada de consideraciones antes expuestas, debemos buscar signos claros de qué hipótesis han sido rechazadas u aceptadas por Platón. Parto de la premisa de que la gimnástica, a la vista de lo que dice Parménides en la parte metodológica, tiene dos claras vertientes, pues consiste en ensayar un camino que, por un lado, puede conducir a la errancia propia de lo que termina en un callejón sin salida. Pero, por otro lado, el sentido de esa metodología es hallar la verdad, que, por tanto, debe encontrarse en la otra opción opuesta a la alternativa de la que se han derivado las «consecuencias imposibles» que se nos han descrito. El lector puede objetar que se trata de una interpretación apriorística, ya que, como hemos visto, algunas interpretaciones consideran inconcluyentes todas las hipótesis, mientras que para otros son todas verdaderas. Vayamos, pues, a los hechos expuestos. En esta ocasión, de los muchos signos que podíamos elegir para adivinar el sentido del texto platónico, propongo atenernos al vocabulario cognitivo que acompaña a la mayoría de las hipótesis ${ }^{19}$.

Examinemos las dos primeras. El carácter contradictorio de las dos primeras hipótesis no es fácil de ver a primera vista, pero si establecemos esquemáticamente lo que afirman y niegan, se pone claramente de manifiesto. La primera, efectivamente, viene a decir que, si el Uno es, entonces no es, por ejemplo, múltiple, mientras que la segunda afirma lo que ha sido negado por la primera y sostiene que, si el Uno es, debe ser múltiple (entre otras muchas

18 Puede verse un esquema muy claro de las ocho en SAYre, K. M., Parmenides' lesson: translation and explication of Plato's Parmenides, University of Notre Dame, Notre Dame, 1996, p. 119 y en Gill, M .L., Philosophos: Plato's Missing Dialogue, p. 49; «Design of the Exercise in Plato's Parmenides», p. 500.

19 Esta parte del trabajo fue debatida en el marco de una ponencia presentada en el XII Symposium Platonicum celebrado en París en julio de 2019. Agradezco mucho las observaciones que me hicieron mis colegas de la International Plato Society. 
cosas). Como ha explicado Miller con toda claridad, a cada paso que se da en la H. (hipótesis) 2, se dice que el Uno es justamente lo que en el paso correlativo de la H.1 se dice que no es ${ }^{20}$. Dentro de las consideraciones, en cierto modo ajenas al análisis ontológico de las tesis objeto de discusión, pero que, a mi juicio, debemos tener presente como indicios apropiados para interpretar el sentido que Platón confiere al valor de verdad de cada hipótesis, está la única expresión en la que los interlocutores valoran los resultados de las diferentes hipótesis: ¿Es posible, pregunta Parménides (142a), que suceda a propósito de lo Uno lo que ha quedado establecido en esta hipótesis (la H.1)? «A mí, al menos, no me lo parece, contesta Aristóteles». No comprendo cómo puede menospreciarse una indicación como esta por parte de aquellos especialistas que, como Meinwald, se han atrevido a afirmar que todas las hipótesis son válidas. Pero hay un segundo indicio de que el Uno, tal y como lo concibe la H.1, no puede ser aceptado. Parménides nos dice que, en tal caso, es decir, si el Uno es absolutamente uno hasta el punto de ser solo uno, no habría para él «ni nombre, ni enunciado, ni

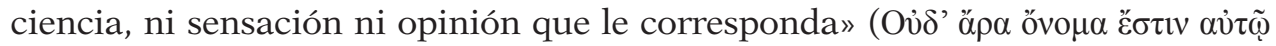

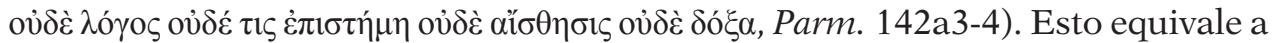
poner de manifiesto que, sobre las bases establecidas en la H.1, no sería posible el discurso ni el pensamiento no ya en el plano epistémico superior de lo inteligible

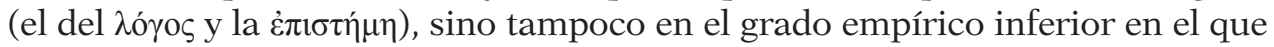

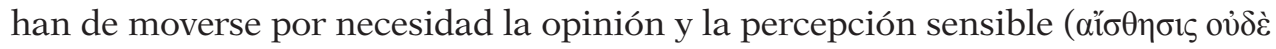
$\delta o ́ \xi \alpha)$. La aparición del vocabulario cognitivo no puede ser, por otra parte, una circunstancia meramente externa a las consideraciones ontológicas, pues, tanto para el pensador de Elea como para el autor del diálogo, el ser y el pensar eran concebidos como dos elementos vinculados en una correlación inseparable. Por otro lado, si reparamos en el contenido ontológico de la hipótesis misma, esta llega a la conclusión de que el Uno no podría ni siquiera ser uno (

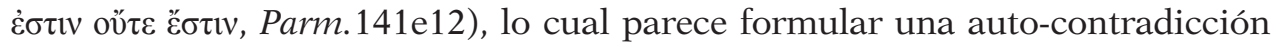
de la que no se ve manera alguna de escapar ${ }^{21}$.

Si ahora nos volvemos a la H.2, que entra en contradicción con la anterior, los resultados, desde el punto de vista del vocabulario cognitivo, son los opuestos a los que acabamos de ver, pues de este Uno, que se relaciona con lo otro de

20 Miller, M. H., Plato's Parmenides: the conversion of the soul, Princeton University Press, Princeton, 1986, p. 84: «Hypotheses I and II stand in pointed contradiction to each other, both as whole positions and at every particular step... Thus the contrast between positive and negative is intensified: the One in II is said to be both/and just what...the One in I is said to be neither/nor». El carácter contradictorio es tal que, a juicio de BERTI (Nuovi studi aristolici, p. 167), «la refutación de la primera lleva consigo la demostración de la segunda».

21 Cfr., en este sentido, SAYre, K. M., Parmenides' lesson: translation and explication of Plato's Parmenides, p. 157: "What Parmenides has shown precisely is that if this one is (H1), then it is not (141e19); and I see no way of reading this that enables it to escape selfcontradiction». Por otro lado, no parece que la distinción entre lo que el Uno es en relación

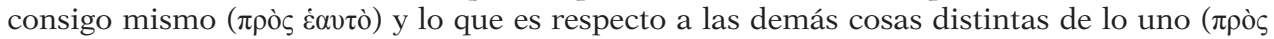
$\tau \tilde{\alpha} \lambda \lambda \alpha$ ), como quiere Meinwald, pueda liberarle de esta contradicción, ya que el Uno, concebido de esta forma, no podría ni siquiera ser uno. 


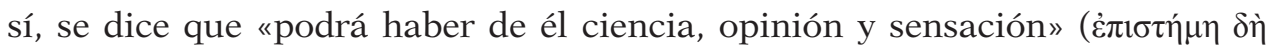

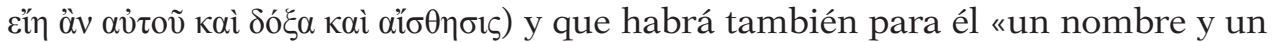

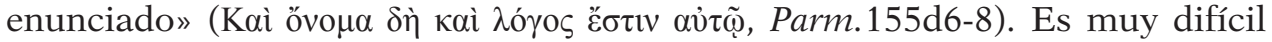
rechazar el valor de verdad que Platón atribuye a esta H.2, como hacen algunos comentaristas $^{22}$, cuando Parménides deja bien claro que el Uno, así concebido, puede ser objeto de ciencia y opinión, contrariamente a lo que se ha dicho en la H.1, que el interlocutor de Parménides ha declarado explícitamente inviable. Por otro lado, se comprende en parte el rechazo que ha suscitado esta hipótesis por parte de los intérpretes, debido a las contradicciones que aparentemente se suscitan en su interior cuando el Uno se relaciona positivamente con lo otro de sí. A este respecto hay que distinguir en mi opinión las contradicciones que se dan entre las hipótesis mutuamente enfrentadas de las contradicciones aparentes que surgen en los caracteres que se atribuyen a lo Uno dentro de ellas. Respecto a estas últimas, como se ha observado alguna vez ${ }^{23}$ y a diferencia de lo que ocurre en otros diálogos, en los que es una indicación de que no se ha argumentado correctamente, los interlocutores no expresan sentimiento alguno de insatisfacción ante este hecho. Por otra parte, en este sentido, desde un punto de vista estrictamente ontológico, ese mismo Sócrates que se muestra impotente para resolver las objeciones de Parménides afirma que sería un portento ( $\tau \dot{\varepsilon} \rho \alpha \varsigma$, Parm. 129b2) si alguien pudiera mostrar que «las cosas que son en sí mismas semejantes se tornan desemejantes o las desemejantes semejantes», lo cual apunta, como se comprueba unas líneas más abajo, al hecho de que las formas, como «la semejanza y la desemejanza, lo múltiple y lo Uno ${ }^{24}$, el reposo y el movimiento», y no solo las cosas del mundo sensible, tienen que admitir en sí mismas «mezclarse y discernirse» (Parm. 129e2-3). Esta limitación de la dialéctica empleada por el joven Sócrates es la que, una vez resuelta, permitirá, siguiendo los pasos de la segunda parte, abrir el camino que conduce a la interrelación de las formas abordada en el Sofista. Sócrates no dice que tal hecho, si pudiera probarse dialécticamente, sea imposible, sino que sería algo admirable y asombroso (Parm. 129e3). Por otro lado, algunas de estas aparentes contradicciones, apuntan a diferentes sentidos en que una forma puede participar de características contrarias u opuestas y debemos indicar, aunque sea de pasada, que, como dijo Cornford, "fue en el Parménides...donde Aristóteles aprendió la máxima que repite tan a menudo de que el Uno y el ser se usan en muchos sentidos» ${ }^{25}$. Desde luego, en los Tópicos el lector puede encontrar todo un capítulo dedicado a esta cuestión, que se concibe como uno de los instrumentos fundamentales de la dialéctica.

Sin embargo, probablemente el argumento más importante a favor de la seriedad y el valor de verdad que Platón concede a la H.2 es, a mi juicio, el

22 Cfr. GILl, M. L., «Design of the Exercise in Plato's Parmenides», p. 503.

23 Cfr. Meinwald, C., Plato's Parmenides, p. 21.

24 Obsérvese en esta relación de formas la presencia de lo Uno, a la que se dedica la segunda parte de la obra.

25 Cornford, F. M., Platón y Parménides, Visor, Madrid, 1989, p. 175. 
Apéndice que se añade a la discusión de las tesis, aparentemente contradictorias, que se han establecido en ella. Aquí, en 155e8-9, Parménides pregunta a su interlocutor si es posible que el uno «en el momento en que participa no participe» o viceversa, que «participe en el momento en que no participa». De la negativa de Aristóteles se concluye, entonces, que «en un tiempo participa y en otro tiempo no participa» y que «este es el único modo en el que podría participar y no participar de lo mismo» (155e10-11). Platón parece enfrentarse con este principio a todos los casos contradictorios que dependen del tiempo y, efectivamente, a este pasaje sigue una reflexión sobre la relación que se establece

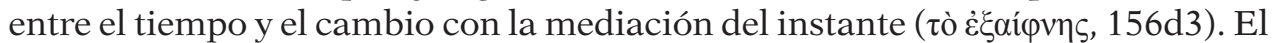
Uno es capaz de participar de los contrarios o experimentar estados opuestos (como el ser y el no ser, la semejanza y la desemejanza, lo pequeño y lo grande, lo igual y lo desigual, el movimiento y el reposo con todos los demás contrarios implicados) por la mediación del instante, que evita una violación del principio de no contradicción. M. L. Gill, que no acepta la viabilidad de la H.2, afirma que «las contradicciones reaparecen en el instante, porque en el instante del cambio lo Uno es tanto no-F como F, ya que (en su opinión), algo que no está en reposo está en movimiento y algo que no está en movimiento está en reposo, de manera que en el instante del cambio lo Uno está tanto en movimiento como en reposo ${ }^{26}$. Sin embargo, en este punto tenemos que distinguir entre los pareceres de algunos intérpretes, a quienes puede legítimamente no satisfacer la solución ofrecida, y lo que el propio Platón nos está diciendo. Lo que Platón dice es que el Uno «cambia en el instante y en el momento en que cambia no podrá hallarse en ningún tiempo» (Parm. 156e5-7). Esta teoría del instante no parece una mera ocurrencia, sino un serio esfuerzo para salvar al Uno de las contradicciones que pueden sobrevenirle cuando interactúa en el devenir. Y un fuerte indicio de ello nos viene proporcionado por Aristóteles. No me refiero ahora al interlocutor de Parménides en la obra, sino al discípulo de Platón, pues el Estagirita declara en la Física que «nada se mueve en el ahora» (VI 3, 234a24; trad. de J. L.Calvo) añadiendo unas líneas más abajo que «tampoco será posible que esté en reposo ${ }^{27}$, lo cual me parece un eco inequívoco de la teoría platónica y un signo evidente de que Aristóteles se la tomó completamente en serio. Aunque Gill suponga que «Parménides permite al Uno violar la ley del tercio excluso en el instante ${ }^{28} \mathrm{y}$, en consecuencia, no acepta que el Apéndice pueda salvar las deficiencias de la H.2, en mi opinión, la doctrina aristotélica, que ubica el ahora entre el reposo y el movimiento, es una muestra muy clara de que se trata de una doctrina pensada con la intención de salvar la contradicción implicada por las propiedades contradictorias que le sobrevienen al sujeto en el proceso del devenir. La claridad con que se impone esta conclusión viene

26 Cfr. Gill, M. L., Philosophos: Plato's Missing Dialogue, p. 65.

27 Esto no significa que no haya diferencias entre la teoría platónica del instante y la concepción aristotélica del ahora; cfr., en este sentido, Allen, R. E., Plato's Parmenides: translation and analysis, University of Minnesota Press, Minneapolis, 1983, p. 265.

28 Gill, M. L., Ryan, P., Parmenides, Hackett, Indianápolis, 1996, p. 86. 
determinada por el mismo Aristóteles cuando este afirma, a propósito de lo que estamos discutiendo, que «el ahora no es principio y fin de lo mismo, pues los contrarios serían simultáneos y, además, con respecto a lo mismo» (Fís. IV 13, 222b5-6). La relación entre el «ahora» ( $\tau$ ò võv) en la terminología aristotélica, el devenir, la atribución de propiedades contradictorias u opuestas y el principio de no contradicción es evidente y a ello apunta, con todas las diferencias que existan, la doctrina platónica del instante $\left(\tau o ̀ ~ s ̇ \xi \alpha i ́(\varphi v \eta \varsigma)^{29}\right.$.

La dialéctica consiste en una práctica que argumenta partiendo de dos hipótesis contradictorias y todo parece indicarnos que la H.1 es un camino inviable, mientras que la H.2, a pesar de las dificultades y el asombro de las aparentes paradojas que pueda suscitar, es la única vía, como hemos visto, transitable para el pensamiento y la opinión. Si ahora examinamos las demás desde este planteamiento, que intenta averiguar el valor de verdad que Platón concedía a las ocho hipótesis partiendo del fuerte indicio proporcionado por la presencia del vocabulario cognitivo, todo parece confirmarnos lo dicho hasta el momento. La H.1, que supuso la existencia de un Uno sin conexión con ninguna otra cosa, tiene su clara correspondencia en la H. $6^{30}$, que pertenece a la serie negativa. El Uno se contempla ahora como algo «que no es de ningún modo ni en ningún sentido ni participa en manera alguna del ser» (Parm. 163c6-7). Al carecer de determinaciones ontológicas (cfr. Parm. 163e7, 164e7), es la contrapartida natural de un discurso puramente negativo que excluye la posibilidad de cualquier acceso cognitivo. Por tanto, la conclusión que demuestra la falsedad e inaceptabilidad de esta hipótesis, a mi juicio, es la imposibilidad de relacionar su objeto con la epistếmē, la dóxa o la aísthēsis, así como con sus correspondientes expresiones lingüísticas (lógos y ónoma, Parm. 164b1-2). Debemos recordar que para el Parménides del diálogo la mayor (mégiston, 133b4) o principal dificultad (aporía) derivada de concebir las formas como propone el joven Sócrates es convertirlas en objetos aislados que no pueden ser conocidos.

Continuemos con el diseño dialéctico del ejercicio para verificar la coherencia de las correspondencias existentes entre las diversas hipótesis. La H.6 se halla «en contradicción directa» con la H.5, como muchos autores han observado $^{31}$, ya que la H.5 afirma (161e3) y la H.6 niega (163c8-d1) que el Uno que no es "participe del ser». La H.5 se basa en el presupuesto de que el Uno que no es debe "participar de algún modo del ser» (161e3), con lo cual también participará «del aquel, del algo, del de este, del para este» y así sucesivamente (160e3-4). Por tanto, estamos en la H.5 ante una ontología que constituye una contradicción de la que se viene a defender en la H.6, según la cual, como

29 Cfr. Berti, E., Nuovi studi aristolici, p. 399: «La prueba más convincente de la no contradictoriedad del instante es el hecho de que la misma doctrina es retomada por Aristóteles, el cual en la Física precisa que el «ahora es la continuidad misma del tiempo, porque enlaza el tiempo transcurrido con el que será sin interrupción alguna».

30 Cfr. SaYre, K. M., o.c., p. 159; Kahn, C., Plato and the post-socratic dialogue: the return to the philosophy of nature, Cambridge University Press, Cambridge, 2013, p. 33.

31 Cfr. Miller, M., o.c., p. 149. 
hemos visto, el Uno que no es «no es de ningún modo ni en ningún sentido ni participa en manera alguna del ser» (163c6-7). Al contrario de lo que se propone en la H.6, las determinaciones positivas de lo Uno que no es permiten que sea «cognoscible» ( $\gamma v \omega \sigma \tau o ́ v, 160 c 7)$ y un objeto de la expresión lingüística

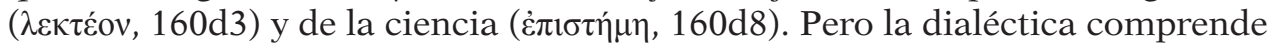
para Platón dos planos que se hallan vinculados necesariamente, la ontología y el conocimiento, de manera que sería absolutamente extraño que estuviera proponiendo una ontología falsa en correspondencia con un plano cognitivo que hace posible el conocimiento y la ciencia, por lo que estamos ante un indicio claro de que la dialéctica ofrece dos opciones contradictorias de las que una debe ser considerada verdadera y la otra falsa, lo cual nos indica que la H.5 debe tener un valor de verdad positivo, coincidiendo con la H.2, mientras que sus contradictorias H6 y H.1, respectivamente, deben ser inadmisibles, ya que estas no son viables cognitiva ni lingüísticamente.

Para terminar, la presencia del vocabulario cognitivo es clara también en las H. 7 y 8, aunque no aparece en la H.3 ni en la H.4. Sin embargo, si examinamos las correspondencias de las tesis ontológicas sostenidas en estos dos pares, de nuevo sus contrapartidas cognitivas constituyen un claro indicio de su viabilidad. Sayre ${ }^{32}$ ha mostrado la convergencia entre la H.3 y la H.7, a pesar de la diferencia existente entre ambas, ya que las tesis ontológicas de la H.7 son interpretadas en términos de meras apariencias. Pero la doctrina formulada en la H.3 en un sentido positivo y en la H.7 en un sentido negativo es la misma: si el Uno existe, entonces las demás cosas admiten (H.3) o, al menos parecen admitir (H.7), todos los caracteres contrarios mencionados en ambos casos (serán limitadas e ilimitadas, semejantes y desemejantes a sí mismas y entre sí, las mismas y diferentes entre sí; cfr.158d-159a para la H.3; 165c-e para la H.7). Aunque en la H.3, no hay el vocabulario cognitivo que hemos venido comentando en el resto de las hipótesis, se nos pide que realicemos lo que algún comentarista ${ }^{33}$ ha llamado «un experimento mental» que exige la intervención de la diánoia para comprender el papel que juega el Uno respecto a la multiplicidad que participa de él. Tenemos en efecto, que realizar «una abstracción mental» ${ }^{34}$ de la función que el Uno desempeña en la constitución de la realidad como mezcla del límite y la pluralidad. Esta referencia explícita a la diánoia (158c2) y "la mención repetida de la visión»35 (ídōmen, 158b8; horômen, 158c6;) revela indirectamente la viabilidad de esta deducción. Sin embargo, si nos cabe alguna duda, hay que tener presente que en la H.7, que ofrece desde una perspectiva negativa la misma versión de la contribución que el Uno presta a los otros, la presencia del vocabulario cognitivo es inconfundible. Aquí se nos recuerda constantemente la importancia del lenguaje (164b7) y la

32 Cfr. Sayre, K. M., Parmenides' lesson: translation and explication of Plato's Parmenides, p. 292.

33 KAHN, C., O.C., pp. 28 y s.

34 SAYRe, K. M., o.c., p. 132.

35 KaHN, C., o.c., p. 29. 
argumentación (logos, 164b8), que representan un punto de partida vinculado esencialmente con las conclusiones ontológicas que constituyen el resultado de esta deducción. Las referencias al papel del pensamiento (diánoia, 165a8, b6, c2) y a las apariencias que requieren la presencia activa del sujeto revelan en qué medida las conclusiones ontológicas están estrechamente unidas a la representación conceptual. Desde luego, a mi juicio, esto es un indicio claro de la viabilidad de estas hipótesis (H.3 y 7), que muestra la contribución del Uno y al mismo tiempo el papel del pensamiento y la percepción en la constitución del mundo de las apariencias.

Por el contrario, dado el carácter contradictorio que tienen las hipótesis H.3 respecto a H.4 y H.7 respecto a H.8, como podíamos suponer, en estas últimas (H.4 y H.8) llegamos a conclusiones opuestas ${ }^{36}$. La H.8 es la contradictoria de la H.7, pues en esta deducción incluso la posibilidad de la mera apariencia es claramente negada y los otros no pueden ser ni parecer unos ni múltiples. La «apariencia de igualdad» ( $\varphi \alpha ́ v \tau \alpha \sigma \mu \alpha$ i $\sigma o ́ \tau \eta \tau o \zeta, 165 a 5)$ y todas esas ilusiones

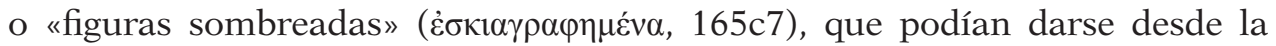
perspectiva de la H.7, desaparecen, efectivamente, en la H.8 y, entonces, ni la

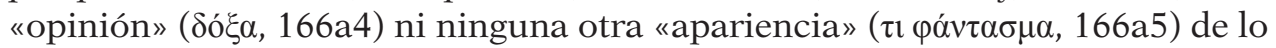
que no es es ya posible. Con lo cual tenemos la confirmación que esperamos, desde el punto de vista del vocabulario cognitivo, sobre la viabilidad o inviabilidad de ambas hipótesis contradictorias, pues en la H.8 se insiste hasta en cuatro ocasiones en la imposibilidad de formular opiniones sobre las bases ontológicas establecidas. La negatividad absoluta ahora defendida, frente a la opción contemplada en la H.7, se revela como un camino tan imposible de seguir para la dialéctica como lo era el no ser al que se refería el Parménides histórico en su poema. Esta exclusión absoluta de lo Uno en su relación con la multiplicidad, desde un punto de vista ontológico, hace inviable cualquier enjuiciamiento o pronunciamiento lingüístico y muestra así la imposibilidad de la hipótesis en sí misma, al partir de un supuesto que hace impracticable cualquier intento de abordar tales resultados por medio del pensamiento.

La viabilidad de la H.3 se revela incompatible con la H.4, ya que ambas extraen consecuencias contradictorias para los otros ${ }^{37}$. En el caso de la H.4, el vocabulario cognitivo está absolutamente ausente como consecuencia directa de la ontología supuesta en esta hipótesis, que afirma que las demás cosas

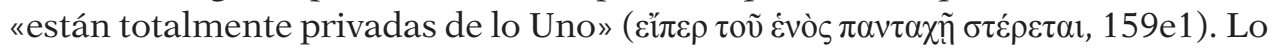
que se desprende de esta tesis es el carácter absolutamente negativo de lo que puede atribuirse a las otras cosas, que no pueden ser «las mismas ni diferentes,

36 El profesor Berti ha insistido en el carácter contradictorio de las hipótesis del Parménides así como en su interpretación desde la concepción aristotélica de la dialéctica, en la que la oposición por contradicción (afirmación y negación de un mismo atributo a un mismo sujeto) implica que, de dos proposiciones opuestas, una es verdadera y la otra falsa. Cfr. «Differenza tra la dialettica socrática e quella platónica» incluido en BerTi, E., Nuovi studi aristotelici, pp. 201-214, esp. pp. 208 y ss.

37 Cfr., p. ej., Miller, M. H., o.c., p. 124. 
ni estar en movimiento ni en reposo...» (160a4). El hecho de que estas cosas diferentes de lo Uno «no puedan tener ninguna otra afección de tal tipo» (oủdè

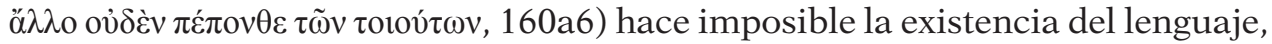
el juicio y el conocimiento. En este caso, la H.4, que las desvincula totalmente de lo Uno, no contiene referencias explícitas al vocabulario cognitivo, que nos está sirviendo de guía indiciaria para comprobar, en este caso, la inviabilidad de esta suposición que se opone contradictoriamente a la H.3. Pero sobre tales bases ontológicas, es evidente que no es posible el lenguaje ni el conocimiento. La confirmación de ello nos llega, sin embargo, de la H.8, que es equivalente de ella en la serie negativa (que niega la existencia de la Uno). Aquí, efectivamente, sí que encontramos explícitamente declaradas las consecuencias cognitivas que se establecen para tal resultado ontológico (166b), derivado, igual que en la H.4, del hecho de que «las otras cosas de ningún modo y en ningún sentido tienen

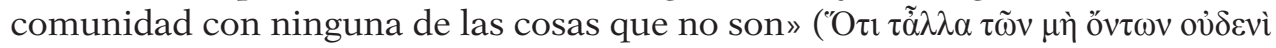

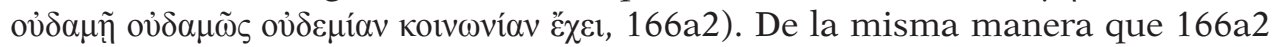
hemos de suponer que ocurre en la H. 4, ahora se afirma explícitamente que sobre estas bases no son posibles la opinión ( $\left.\delta \delta^{\xi} \xi \alpha\right)$ ni la apariencia ( $\varphi \alpha ́ v \tau \alpha \sigma \mu \alpha$, 166a4-5). Dado que lo uno no es y se excluye de forma absoluta cualquier participación de la multiplicidad de los otros en ello, Parménides tiene que declarar que los otros y la multiplicidad no pueden ser concebidos ni objetos

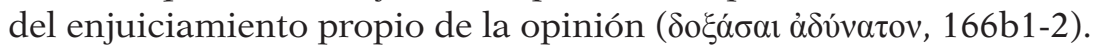

\section{CONCLUSIÓN}

Aunque no he entrado de lleno en la discusión del contenido ontológico de cada hipótesis, en medio de la incertidumbre de los intérpretes, los caminos de la dialéctica parecen claramente esclarecidos a partir de ese triángulo de ideas establecido en la parte metodológica del diálogo. Se trata de «recorrer todos los caminos» en una concepción gimnástica de la dialéctica que refleja el verdadero proceso del pensamiento, el cual no es tan sencillo como podíamos suponer desde una interpretación demasiado «científica» o estrecha de sus procedimientos. Esta concepción de la dialéctica refleja el movimiento del pensamiento filosófico, que tiene que confrontar diversas posibilidades, como muestran, por otro lado, las mismas controversias filosóficas que se han pronunciado acerca de lo Uno y lo múltiple. Pero, en segundo lugar, hay caminos que, aunque hayan sido intentados por otros filósofos, nos conducen a una «errancia» que se manifiesta en «la imposibilidad» o la inviabilidad de sus resultados. Esto es lo que la exploración del vocabulario cognitivo ha puesto de manifiesto, tanto desde un punto de vista negativo como positivo. Está claro que allí donde no es posible el lenguaje y la opinión, ni la ciencia o el pensamiento, se ha incurrido en la suposición de una ontología imposible. Cuando se afirma, por el contrario, la posibilidad de la ciencia y el lenguaje, no puede haberse formulado una ontología desechable, sino principios necesarios 
para repensar la naturaleza de unas ideas que el joven e inexperto Sócrates no ha sabido defender, - y estos principios resurgirán en diálogos posteriores para repensar la interrelación entre las ideas, precisamente como un requisito ontológico que hace posible el pensamiento del ser. Pero, en tercer lugar, no se trata de un ejercicio o de una actividad meramente lúdica que el entendimiento acometa con la frivolidad sofística de un pensamiento que se limita a jugar con las ideas y las palabras, sino de una técnica de la argumentación que está al servicio de la verdad, porque se afirma solo después de confrontarse con los caminos errados del pensamiento. El interés del Parménides, a diferencia del programa aparentemente cientificista de la República, radica en que refleja el movimiento verdadero del pensar y su naturaleza dialógica, que se aventura en busca de la verdad por los caminos empedrados de las aporías y las controversias filosóficas.

Universidad de Granada

Álvaro Vallejo Campos

avallejo@ugr.es

[Artículo aprobado para publicación en febrero de 2021] 